\title{
Tunable Fabry-Perot Interferometer Based on Fiber Bragg Gratings
}

\author{
Islam Ashry \\ The Bradley Department of Electrical and Computer \\ Engineering. \\ College of Engineering, Virginia Tech. University. \\ Virginia, USA. \\ ashry@vt.edu.
}

\author{
Hossam M. H. Shalaby \\ Department of Electrical Engineering. \\ Faculty of Engineering, University of Alexandria. \\ Alexandria, Egypt. \\ shalaby@ieee.org.
}

\begin{abstract}
In this paper, we propose a new architecture for tunable Fabry-Perot (FP) interferometer using fiber Bragg gratings (FBGs). The main feature of the architecture is its ability to increase the finesse using a potentially low cost technique. Furthermore, it decreases the power loss of the filtered channel and can be implemented easily in practice.
\end{abstract}

Keywords-Fabry-Perot (FP); filter; fiber Bragg grating(FBG); wavelength-division multiplexing (WDM).

\section{INTRODUCTION}

Over the past few years, the networks traffic has grown rapidly and the optical transport bandwidth has been continuously increasing. Optical networking using wavelength-division multiplexing (WDM) is the technology of choice for meeting these growing demands. Since in WDM systems each channel is related to a different wavelength, channel manipulations and particularly channel selection require optical wavelength selection (i.e., optical filtering). Single and multiple tunable Fabry-Perot (FP) interferometers have long been used as narrow-band commercial optical filters for WDM networks [1-3].

Most of conventional FP interferometers have the following limitations: Firstly, the maximum number of channels that can be filtered, finesse, is limited. Increasing this number requires using dielectric mirrors of high reflectivity, which costs a lot $[4,5]$. Secondly, through the reflection of light in the resonant cavity by the two mirrors, light is coupled in and out of the optical fiber which yields to power loss [4].

In this paper, we propose a novel design to a tunable FP interferometer based on fiber Bragg gratings (FBGs). The main advantage of the proposed architecture over the conventional tunable FP interferometers is its ability to provide high finesse with potentially low cost. In addition, using FBGs instead of mirrors in the resonant cavity guarantees that the light is always inside the optical fiber. This property, of course, is very effective to decrease the power loss of the filtered channel. Furthermore, since FBGs provide all-fiber geometry, the proposed architecture is easy to be fabricated.

\section{DESCRIPTION OF THE PROPOSED TUNABLE FIBER FABRY-PEROT FILTER}

Fig. 1 shows the physical configuration of the proposed fiber FP tunable filter. This architecture is mainly composed of a single-mode optical fiber of length $z$. This fiber is terminated by two groups of FBGs. Each group has $N$ FBGs of Bragg wavelengths $\lambda_{1}, \lambda_{2}, \ldots, \lambda_{N}$. Also, $\lambda_{1}, \lambda_{2}, \ldots, \lambda_{N}$ are the equally spaced wavelengths used in the network. The distance between two consecutive FBGs in the same group is $y$ and between two FBGs of the same Bragg wavelengths, one in the first group and the other in the second group, is $x$. These FBGs play the same role of mirrors in the conventional FP filter, but each FBG is responsible for reflecting only single wavelength. The used FBGs are characterized by a reflectivity, $R$, and out of band transmission loss, $A$.

The resonant frequency of this device can be changed by adjusting the length of the single-mode fiber, $z$. This length can be changed by using either thermo-optic control mechanisms [6].

Fig. 2 illustrates the basic operation of the proposed tunable FP filter and gives the strength of the electric field, $E$, inside it. Let, for example, light of field strength $E_{i}$ and wavelength $\lambda_{1}$ enters the filter and passes through the first FBG in the first group, $E_{i} \sqrt{1-R}$ is transmitted, and the rest is reflected to the left from the device. The signal then continues to pass through the remaining FBGs in the first group and leaves the last FBG in the first group with field strength of $E_{i} \sqrt{1-R}(1-A)^{\frac{N-1}{2}} e^{-j k(N-1) y}$, where $k=2 \pi n / \lambda_{1}$ is the propagation constant and $n$ is the core refractive index of the single-mode fiber. When the signal passes through the first FBG in the second group, a portion of field strength $E_{i} \sqrt{1-R} \sqrt{R}(1-A)^{\frac{N-1}{2}} e^{-j k x}$ is reflected back toward the left, while the rest of field strength $E_{i}(1-R)(1-A)^{\frac{N-1}{2}} e^{-j k x}$ continues to propagate to the right. Finally, the signal leaves the last FBG in the second group with strength of $E_{i}(1-R)(1-A)^{(N-1)} e^{-j k[x+(N-1) y]}$. The rest description of the field strength is shown in Fig. 2.

Adding up all the successive contributions to the output, the output electric field strength from the device, $E_{o}$, can be written as follows:

$$
\begin{aligned}
& E_{o}=E_{i}\left[(1-R)(1-A)^{(N-1)} e^{-j k[x+(N-1) y]}\right. \\
& +(1-R) R(1-A)^{2(N-1)} e^{-j k[3 x+(N-1) y]} \\
& \left.+(1-R) R^{2}(1-A)^{3(N-1)} e^{-j k[5 x+(N-1) y]}+\ldots \ldots . .\right]
\end{aligned}
$$




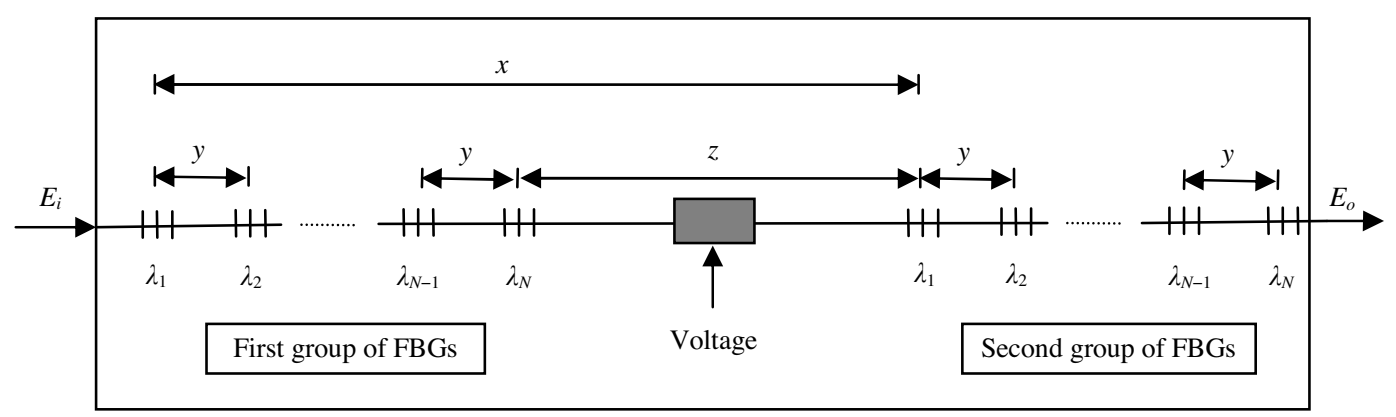

Figure 1. Schematic of the proposed architecture.

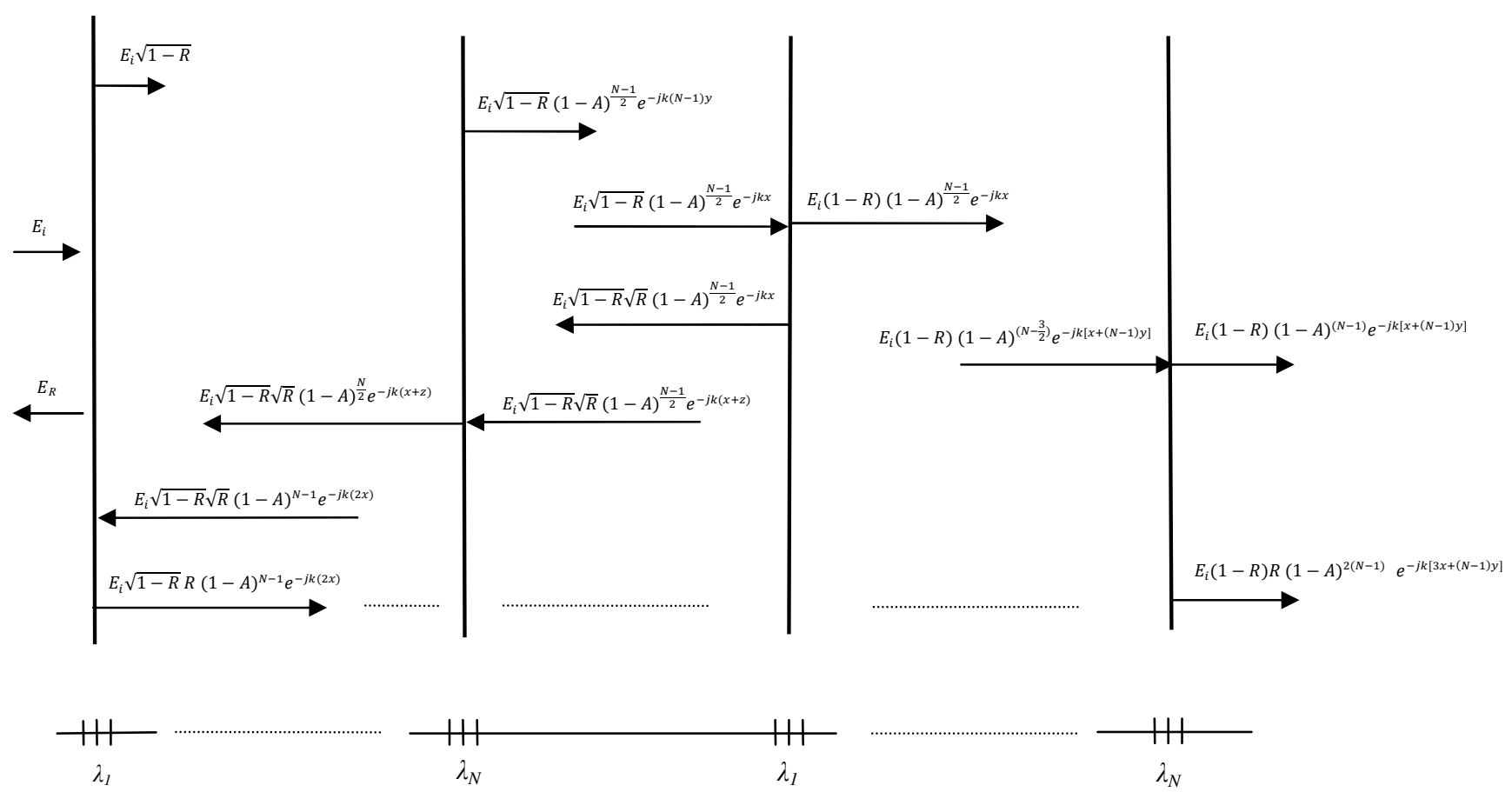

Figure 2. Electric field strength of successive reflections.

The transfer function, $H(f)$, is given by:

$$
H(f)=\frac{E_{o}}{E_{i}}=\frac{(1-R)(1-A)^{(N-1)} e^{-j k[x+(N-1) y]}}{1-R(1-A)^{(N-1)} e^{-j 2 k x}} .
$$

The transmittance, $T(f)$, which is the ratio between output power to incident power, is given by:

$T(f)=|H(f)|^{2}=\frac{(1-R)^{2}(1-A)^{2(N-1)}}{1+R^{2}(1-A)^{2(N-1)}-2 R(1-A)^{(N-1)} \cos (2 k x)}$.

Equation (3) shows that, $T(f)$ is a periodic function with a period called free spectral range (FSR) given by:

$$
F S R=\frac{c}{2 n x} \text {. }
$$

In a WDM network, we could place $N$ equally spaced frequencies within one FSR.

The peak value of transmittance, $T(f)_{\max }$, which occurs at resonance is:

$$
T(f)_{\max }=\frac{(1-R)^{2}(1-A)^{2(N-1)}}{\left[1-R(1-A)^{(N-1)}\right]^{2}}
$$

$T(f)_{\max }$ occurs at $f=m \frac{c}{2 n x}=m . F S R, m \in\{0, \mp 1, \mp 2, \ldots .$.$\} .$ The minimum value of transmittance, $T(f)_{\min }$, is given by:

$$
T(f)_{\min }=\frac{(1-R)^{2}(1-A)^{2(N-1)}}{\left[1+R(1-A)^{(N-1)}\right]^{2}}
$$

The 3-dB bandwidth, called full width at half maximum $(F W H M)$ can be obtained as follows:

$$
\begin{gathered}
T\left(f_{0}\right)=\frac{(1-R)^{2}(1-A)^{2(N-1)}}{1+R^{2}(1-A)^{2(N-1)}-2 R(1-A)^{(N-1)} \cos \left(\frac{4 \pi n x}{c} f_{0}\right)} \\
=\frac{1}{2} \frac{(1-R)^{2}(1-A)^{2(N-1)}}{\left[1-R(1-A)^{(N-1)}\right]^{2}}
\end{gathered}
$$

But $f_{0}$ is relatively very small, therefore (7) can be 
approximated as:

$$
\begin{gathered}
T\left(f_{0}\right) \approx \frac{(1-R)^{2}(1-A)^{2(N-1)}}{1+R^{2}(1-A)^{2(N-1)}-2 R(1-A)^{(N-1)}\left[1-0.5\left(\frac{4 \pi n x}{c} f_{0}\right)^{2}\right]} \\
=\frac{1}{2} \frac{(1-R)^{2}(1-A)^{2(N-1)}}{\left[1-R(1-A)^{(N-1)}\right]^{2}}
\end{gathered}
$$

Solving (8), $f_{0}$ is given by:

$$
f_{0}=\frac{c}{4 \pi n x} \frac{1-R(1-A)^{(N-1)}}{\sqrt{R}(1-A)^{\frac{N-1}{2}}}
$$

Hence,

$$
F W H M=2 f_{0}=\frac{c}{2 \pi n x} \frac{1-R(1-A)^{(N-1)}}{\sqrt{R}(1-A)^{\frac{N-1}{2}}}
$$

The finesse, $F$, is defined as the ratio of FSR to FWHM:

$$
F=\frac{F S R}{F W H M}=\frac{\pi \sqrt{R}(1-A)^{\frac{N-1}{2}}}{1-R(1-A)^{(N-1)}}
$$

\section{RESULTS AND DISCUSSION}

Equation (11) gives an important result that the finesse, $F$, depends on the number of used wavelengths in the network, $N$. In order to select only one wavelength, $F$ should always be greater than or equal to $N$. Consequently, the maximum number of wavelengths, $N_{\max }$, that can be used in the network can be calculated by substituting $F=N=N_{\max }$ in (11):

$$
N_{\text {max }}=\frac{\pi \sqrt{R}(1-A)^{\frac{N_{\max }-1}{2}}}{1-R(1-A)^{\left(N_{\max }-1\right)}}
$$

Since $A$ is relatively very small, one can use the following approximations:

$$
(1-A)^{\frac{N_{\max }-1}{2}} \approx 1-\left(\frac{N_{\max }-1}{2}\right) A
$$

and,

$$
(1-A)^{\left(N_{\max }-1\right)} \approx 1-\left(N_{\max }-1\right) A
$$

Using (13) and (14) in (12), we get a quadratic equation of $N_{\max }$. Solving it, we obtain the following approximate formula of $N_{\max }$ :

$N_{\text {max }} \approx \frac{-2-A \pi \sqrt{R}+2 R(1+A)+\sqrt{8 A(2+A) \pi R^{1.5}+(2+A \pi \sqrt{R}-2 R-2 A R)^{2}}}{4 A R}$

Fig. 3 shows the exact change of $N_{\max }$, using (12), and its approximate change, using (15), with $A$ at $R=99.7 \%$. Similarly, its change is illustrated in Fig. 4 at $R=97 \%$. Obviously, both Fig. 3 and Fig. 4 assert that as $A$ decreases, $N_{\max }$ increases. Also, at constant $A, N_{\max }$ increases with the increase of $R$ and vice versa.

From (12), $N_{\max }$ equals 49 at $R=99.7 \%$ and $A=1.25 \times 10^{-3}$. Using these values, the behavior of the transmittance against the normalized frequency, $f / F S R$, can be illustrated in Fig. 5. Similarly, Fig. 6 shows the change of the transmittance with the normalized frequency at $N_{\max }=35$, $R=99.7 \%$ and $A=2.5 \times 10^{-3}$.

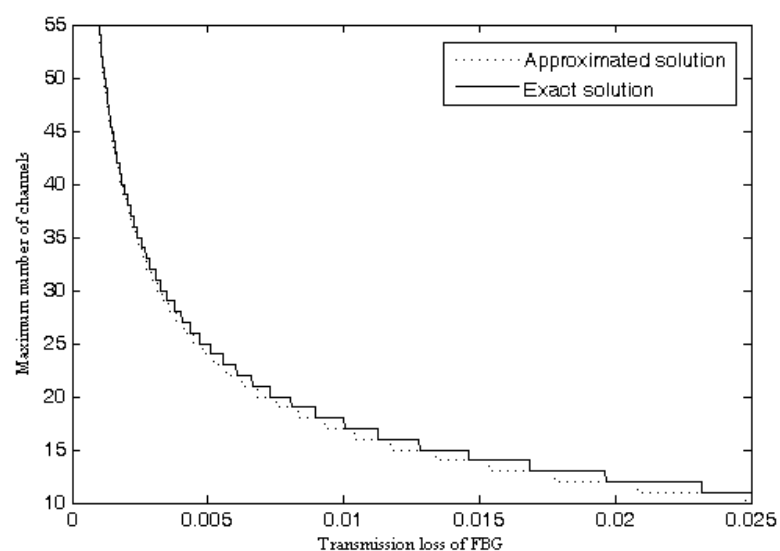

Figure 3. Change of $N_{\max }$ with $A$ at $R=99.7 \%$.

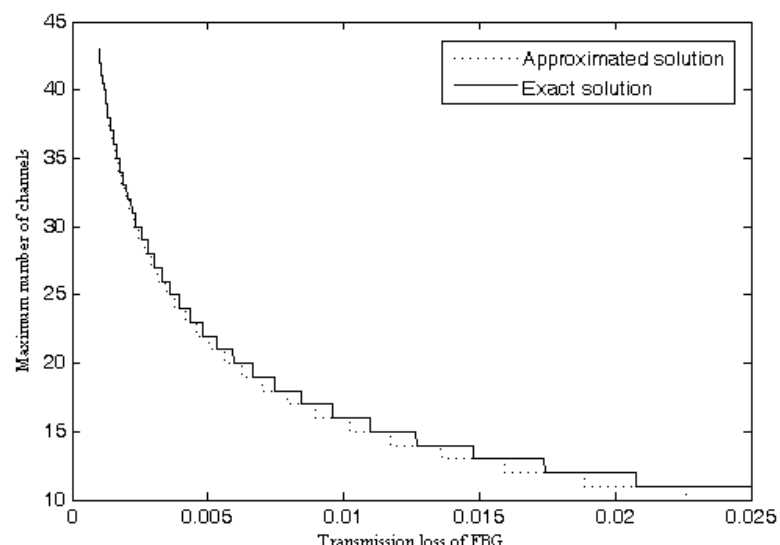

Figure 4. Change of $N_{\max }$ with $A$ at $R=97 \%$.

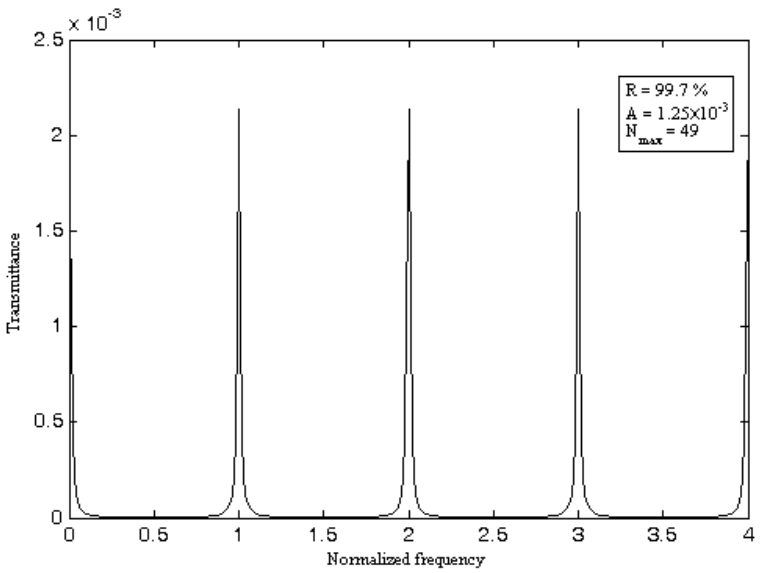

Figure 5. Transmittance of the proposed tunable FP filter at $R=99.7 \%$, $A=1.25 \times 10^{-3}$, and $N_{\max }=49$.

All the obtained results in the previous figures and equations show that, the best performance of the proposed tunable FP filter occurs when $A$ of the used FBGs approaches zero. This yields to increase both $N_{\max }$ and $T(f)_{\max }$. If the technology provides zero out of band transmission loss and $R=99.7 \%$ for the used FBGs, $N_{\max }$ will equal 1045 and $T(f)_{\max }$ reaches unity. 


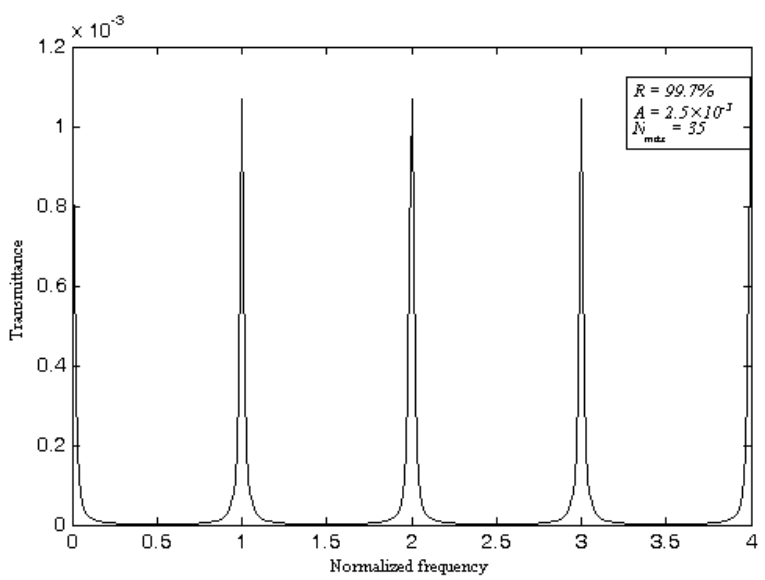

Figure 6. Transmittance of the proposed tunable FP filter at $R=99.7 \%$, $A=2.5 \times 10^{-3}$, and $N_{\max }=35$.

\section{CONCLUSION}

We proposed a novel tunable FP interferometer based on FBGs. The proposed architecture can be used to increase the finesse with a very low cost. Theoretically, a finesse equals 1045 is achievable at zero out of band transmission loss and $R=99.7 \%$ for the used FBGs. Moreover, the proposed system is all embedded in a single-mode optical fiber. Consequently, it cancels the power loss of the filtered channel found in the conventional tunable FP which uses mirrors for reflection. Finally, since FBGs provide all-fiber geometry, this makes the fabrication process of the proposed filter easy.

\section{REFERENCES}

[1] Anatoly Frenkel, and Chinlon Lin, "Angle-tuned etalon filters for optical channel selection in high density wavelength division multiplexed systems," J. Lightwave Technol. , vol. 7, pp. 529-551, April 1989.

[2] Dan Sadot, and Efraim Boimovich, "Tunable optical filters for dense WDM networks," IEEE Communications Magazine , vol. 36, pp. 5055, December 1998.

[3] M. Ogusu, Y. Shimomura, and S. Ohshima, "A thermally stable Fabry-Perot tunable filter for $1 \AA$ spaced high-density WDM systems," IEEE Photonic Technology Letter, vol. 5, pp. 1222-1224, October 1993.

[4] J. Stone, and L. W. Stulz, "Pigtailed high-finesse tunable fibre FabryPerot interferometers with large, medium and small free spectral ranges," Electronics letters. , vol. 23, pp. 781-783, July 1987.

[5] R. P. Netterfield, C. H. Freund, J. A. Seckold, and C. J. Walsh, "Design of a lithium niobate Fabry-Perot etalon-based spectrometer," APPLIED OPTICS , vol. 36, pp. 4556-4561, July 1997.

[6] Sung-Sik Yun, and Jong-Hyun Lee, "A micromachined in-plane tunable optical filter using the thermo-optic effect of crystalline silicon ," J. of micromechanics and microengineering, vol. 13, pp. 4556-4561, September 2003. 